\title{
ORIGINAL ARTICLE International Spinal Cord Injury: Spinal Interventions and Surgical Procedures Basic Data Set
}

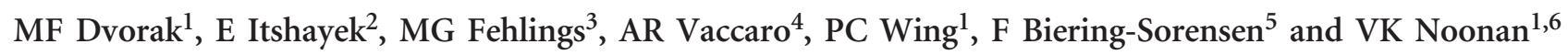

Study design: Survey of expert opinion, feedback and final consensus.

Objective: To describe the development and the variables included in the International Spinal Cord Injury (SCI) Spinal Interventions and Surgical Procedures Basic Data set.

Setting: International working group.

Methods: A committee of experts was established to select and define data elements. The data set was then disseminated to the appropriate committees and organizations for comments. All suggested revisions were considered and both the International Spinal Cord Society and the American Spinal Injury Association endorsed the final version.

Results: The data set consists of nine variables: (1) Intervention/Procedure Date and start time (2) Non-surgical bed rest and external immobilization, (3) Spinal intervention-closed manipulation and/or reduction of spinal elements, (4) Surgical procedure-approach, (5) Date and time of the completion of the intervention or surgical closure; (6) Surgical procedure-open reduction, (7) Surgical procedure —direct decompression of neural elements, and (8 and 9) Surgical procedure —stabilization and fusion (spinal segment number and level). All variables are coded using numbers or characters. Each spinal intervention and procedure is coded (variables 1 through 7 ) and the spinal segment level is described (variables 8 and 9). Sample clinical cases were developed to illustrate how to complete it.

Conclusion: The International SCl Spinal Interventions and Surgical Procedures Basic Data Set was developed to facilitate comparisons of spinal interventions and surgical procedures among studies, centers and countries.

Spinal Cord (2015) 53, 155-165; doi:10.1038/sc.2014.182; published online 25 November 2014

\section{INTRODUCTION}

The treatment of acute traumatic spinal cord injury (SCI) involves performing therapeutic interventions with the goals of minimizing further neurological deterioration and promoting neurological recovery. The ultimate objective is to create a spinal column that is mechanically stable, not a source of pain, and resistant to further neurological injury or deformity over time.

In some cases, this can be achieved without open surgical procedures; that is, without general anesthesia and without a surgical incision that exposes the spinal elements. ${ }^{1-3}$ In other situations, open surgical stabilization and decompression techniques may be the preferred approach to achieving the treatment goals described above $^{4-7}$ The authors recognize that there are unique clinical environments, variations in the injury pattern itself, the presence and absence of associated non-spinal injuries, as well as variability in not only surgeon training and local practice but also in the available resources that will impact and direct treatment decisions. It is not the purpose of this project to specify indications for treatment or even what may or may not be the preferred techniques of treatment. We acknowledge that the ultimate therapeutic objectives may be achieved through the use of various techniques. This project attempts to standardize the nomenclature used to describe basic components of interventions and procedures that may influence the patients' outcome in a manner consistent with the principles of the International SCI Data Set project. ${ }^{8}$

Spinal interventions may involve the application of skeletal traction by means of skull tongs or halo rings of varying design. ${ }^{9,10}$ Interventions may also involve manipulations and closed reduction maneuvers that reduce or realign fractures, dislocations or subluxations. ${ }^{11-13}$ These reductions and manipulations may occur over several minutes, hours or even days and may require varying degrees of anesthesia from local, intravenous sedation to a full general anesthetic. Spinal interventions also include the application of external braces, casts, halo vests and other forms of external immobilization. ${ }^{14,15}$ Prolonged bed rest, which is prescribed as a specific therapy to enable fractures to heal, the spine to stabilize or to facilitate natural neurological recovery, is also a specific spinal intervention that is applied over days or even weeks and months. ${ }^{3,16,17}$

Surgical procedures in all cases require some form of anesthesia, most commonly a general anesthetic, but occasionally procedures may be performed under local anesthesia. Surgical procedures always involve an incision in the skin through which the spinal elements are accessed either with direct visualization or percutaneously. Surgical procedures also encompass the more recently described techniques of percutaneous insertion of screws and rods for stabilization. ${ }^{18}$ Generally, surgical procedures are performed through either anterior

${ }^{1}$ Department of Orthopaedics, University of British Columbia, Vancouver, British Columbia, Canada; ${ }^{2}$ Hadassah-Hebrew University Medical Centre, Jerusalem, Israel; ${ }^{3}$ Department of Surgery, University of Toronto, Toronto, Ontario, Canada; ${ }^{4}$ Department of Orthopaedics, Thomas Jefferson University, Philadelphia, PA, USA; ${ }^{5}$ Glostrup Hospital, University of Copenhagen, Copenhagen, Denmark and ${ }^{6}$ Rick Hansen Institute (RHI), Vancouver, British Columbia, Canada 
or posterior surgical approaches. Posterior approaches include midline incisions as well as minimally invasive, muscle splitting or 'Wiltse'type paraspinal incisions. ${ }^{18}$ Posterior approaches always visualize or access the posterior elements (lamina, facets, transverse processes) although they may be used to access the anterior spinal elements (postero-lateral discectomy or corpectomy). Anterior surgical approaches encompass all anterior and lateral approaches in the cervical, thoracic and lumbo-sacral spine. All anterior approaches will visualize the vertebral body and/or disk but do not enable visualization or direct manipulation of the lamina, facets or other posterior spinal elements. ${ }^{19-21}$

Spinal interventions and surgical procedures will generally achieve their treatment goals by means of the following three steps:

1. Reduction and realignment of displaced vertebrae;

2. Direct or indirect decompression of neural elements; and

3. Stabilization of vertebral elements.

It is not our goal to debate the relative merits and indications for the need for reduction, decompression or stabilization; however, we simply propose that these are the relevant goals of most therapeutic interventions and procedures. There is considerable overlap between the above three steps, and one or more of these steps may be achieved by means of a single open surgical procedure or alternately through one or various therapeutic interventions that do not require general anesthesia or an open surgical incision. At one specific date, a patient may have a combination of interventions and procedures; for example, an open surgical procedure may also involve an external brace to be worn after the surgery. An attempted closed reduction of vertebral elements (intervention) that is not successful may be followed on the same day by an open surgical procedure. ${ }^{22}$ Given these considerations and the increasing need to use common data elements by funding agencies to improve data quality and comparability among studies, the purpose of this project was to develop a minimal data set to describe spinal interventions and surgical procedures that could be used internationally. This data set is referred to as the International SCI Spinal Interventions and Surgical Procedures Basic Data Set.

\section{MATERIALS AND METHODS}

In accordance with the goal of the International SCI Data Set project, the aim of the International SCI Spinal Interventions and Surgical Procedures Basic Data Set is to standardize the collection and reporting of a minimal amount of clinically relevant information about the interventions and procedures performed on individuals with spinal column and SCIs. ${ }^{8,23-26}$ The International SCI Spinal Interventions and Surgical Procedures Basic Data Set is applicable to adult (skeletally mature) and pediatric (skeletally immature) individuals with traumatic supraconal or conal SCI or cauda equina injuries. It is intended to be used with the International SCI Spinal Column Injury Basic Data Set and the International SCI Core Data Set. ${ }^{24,26}$

The first draft of the International SCI Spinal Interventions and Surgical Procedures Basic Data Set was created by a working group consisting of members selected by the group's chair based on their expertise in the field of spinal column injury research, together with representatives of the Executive Committee of the International SCI Standards and Data Sets. The data set was developed with the intent that it could be used internationally, without great expense, and that it would encompass the heterogeneity of clinical practice seen in countries around the world. The development process for the International SCI Spinal Intervention and Surgical Procedures Basic Data Set involved the following steps:

(1) The working group developed a first draft data set in 2012. This was further refined through an iterative process involving all the group members.
A syllabus was developed to provide detailed definitions for each specific variable and response category.

(2) Members of the Executive Committee of the International SCI Standards and Data Sets project reviewed the draft data set and its syllabus.

(3) Comments from the Executive Committee members were discussed among the working group via e-mail and appropriate changes were made to the data set.

(4) Relevant and interested international scientific professional organizations and societies as well as interested individuals reviewed the data set.

(5) The working group reviewed comments from the International community, and changes were recommended and implemented.

(6) Members of the ISCoS Scientific Committee and the ASIA Board reviewed the data set.

(7) The working group discussed comments from the Committee/Board members, and further adjustments to the data set were made.

(8) The data set was finalized in 2014

(9) Standard naming conventions for the variables of the data set were completed in collaboration with the National Institute of Neurological Diseases and Stroke/National Institutes of Health Common Data Element project. $^{27}$

\section{RESULTS}

The International SCI Spinal Interventions and Surgical Procedures Basic Data Set is structured according to the established protocol pertaining to International SCI Data Sets. ${ }^{27}$ The data set is included in Supplementary Appendix S1 and is currently available on the ISCoS website. ${ }^{27}$ A glossary with all procedures recorded using this form is included in Supplementary Appendix S2.

There are nine variables in the data set, and they may be applied and filled out at any time following the injury. Each variable in the data set is described below. Four sample cases are provided to describe how the data set is coded (see Figures 1-4).

\section{Intervention/procedure date and start time}

This variable documents the date and time on which an intervention or procedure was started. When a surgical procedure is initiated on a specific day, that date is specified and then all subsequent aspects of that procedure are recorded for that date. The time that the surgical procedure begins, that is, the time that the initial skin incision is made, is recorded as the Intervention/Procedure time. The time component of this variable documents the time of the surgical incision using a 24-h clock. If part of the information is known (hour but not minute), then this can be entered. The data element 'Unknown' is assigned when no information is available. For non-surgical interventions such as traction, closed manipulations or prescribed bed rest, the time data element may be left unknown as it may not be necessary.

If an intervention lasts for $>1$ day, such as traction or bed rest, then the date that the interventions were initiated is recorded and the time need not be specified. Furthermore, when several interventions or procedures are all performed or initiated on one date, then a single date is entered and all other variables are completed for that specific date. When multiple procedures are performed on separate dates, then these separate interventions and procedures are recorded on individual forms using separate dates that serve as the procedure identifier.

\section{Non-surgical—bed rest and external immobilization}

This variable documents whether there was any non-surgical intervention initiated on the date identified above. This includes prescriptive bed rest, which usually involves $>10$ days of enforced bed rest, and not including the normal recumbency, which follows an injury or surgery as a patient is slowly mobilized. ${ }^{3}$ The application of 

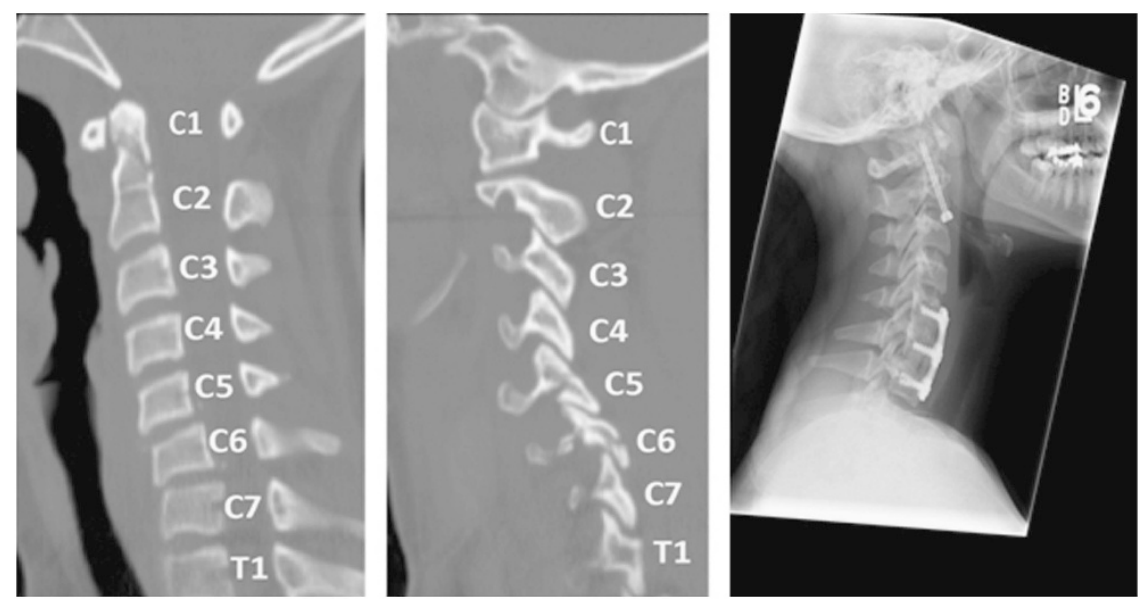

Figure 1 This patient has sustained multiple closed injuries of the cervical spine. In addition to the Type II odontoid fracture, there are fractures of the C5 and C6 facet joints with a subluxation at both the C5-6 and C6-7 levels. Through a single anterior incision (started 6 February 2010 at 1630 hours and finished at 2245 hours), this patient underwent anterior stabilization of the C2 fracture with screw fixation and anterior discectomies; direct decompression by means of the resection of the posterior longitudinal ligament and posterior annulus; and stabilization from vC5 to vC7. The patient afterwards wore an external orthosis for 6 weeks. This would be coded as shown in Appendix A.
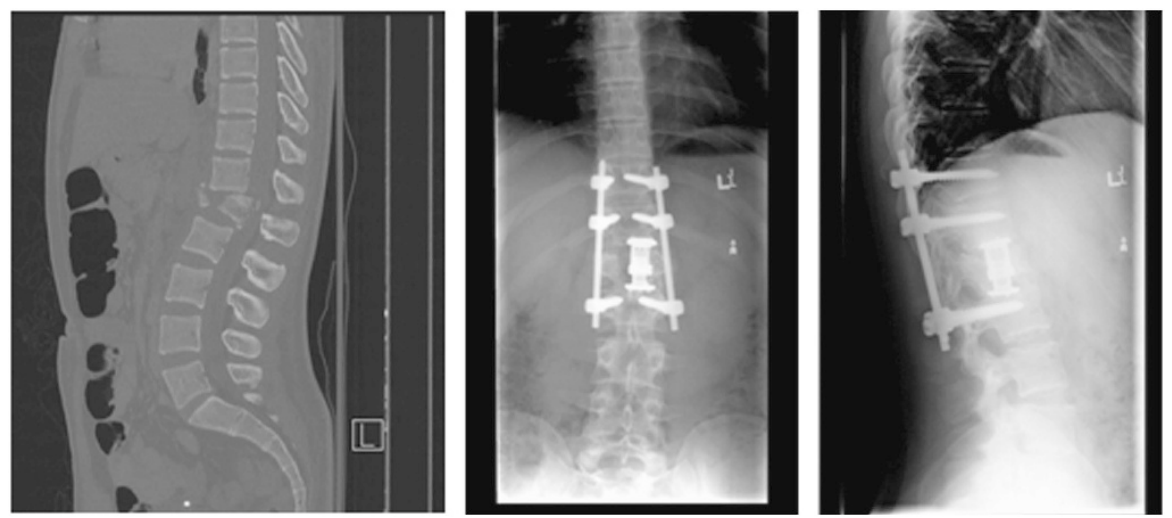

Figure 2 This patient has sustained an L1 burst fracture without ligament injury. The fracture was stabilized (started 26 June 2011 at 2300 hours and ended the following day at 0430 hours) through a posterior approach with fixation between vT11 and vL2 and then a subsequent anterior vertebral body resection (corpectomy) with direct decompression of the neural elements. Anterior stabilization with a cage was performed. No bed rest or external immobilization was prescribed. This would be coded as shown in Appendix B.

external spinal immobilization devices such as braces, casts, halo thoracic vests and so on is also a specific spine intervention.

The code 'No' is assigned to indicate that this patient has not been prescribed bed rest nor has any external immobilization been utilized. 'Enforced bed rest' is used to represent enforced bed rest that is specifically prescribed to either allow fractures to heal or neurological impairment to improve over a time $>10$ days. 'External immobilizing device' code indicates that an external immobilizing device has been applied and this includes body casts, external braces or halo thoracic vests, and 'Both enforced bed rest and external immobilizing device' code indicates that the patient has been prescribed both bed rest and external immobilization. When there is no information regarding non-surgical interventions, 'Unknown' is assigned.

\section{Spinal intervention-closed manipulation and/or reduction of} spinal elements

This variable documents whether closed manipulation and/or reduction of displaced spinal elements has been attempted regardless of whether or not it has been successful. Performance of a closed reduction or manipulation may require application of pins, tongs, head halters or various other forms of traction and subsequent manipulation. This may be performed over several hours or the patient may be left in traction for several days in an attempt to slowly effect a reduction.

The code 'No' is assigned to indicate that no attempt has been made to perform a closed manipulation or reduction of vertebral elements. When a closed reduction or manipulation has been attempted or performed successfully, 'Yes' is assigned. 'Unknown' is selected when the presence or absence of a manipulation or reduction attempt is not known.

\section{Surgical procedure-approach}

This variable documents whether the open surgical procedure was performed by means of an anterior or posterior surgical approach or both. Anterior surgical approaches include all anterior and lateral cervical approaches as well as any thoracic or lumbar approach that 

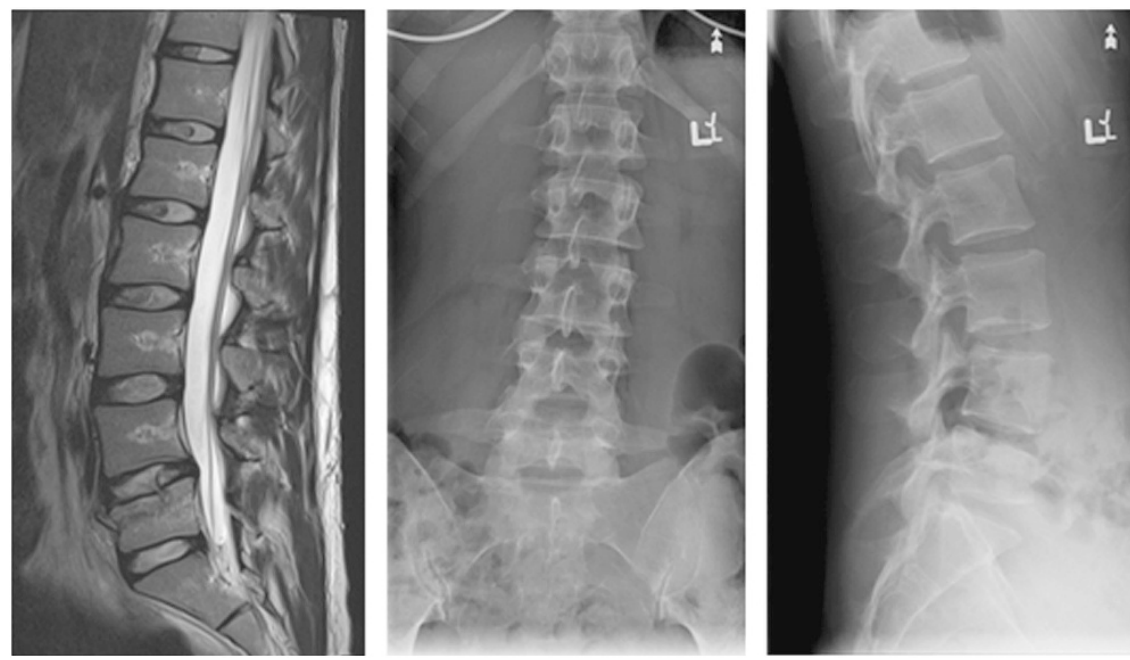

Figure 3 This female fell on 15 August 2012 off a balcony landing on her buttocks. She sustained an L5 burst fracture and the same day was treated with bed rest until 5 September 2012 and then gradual mobilization without a brace or orthosis. She did not have surgery. This would be coded as shown in Appendix C.
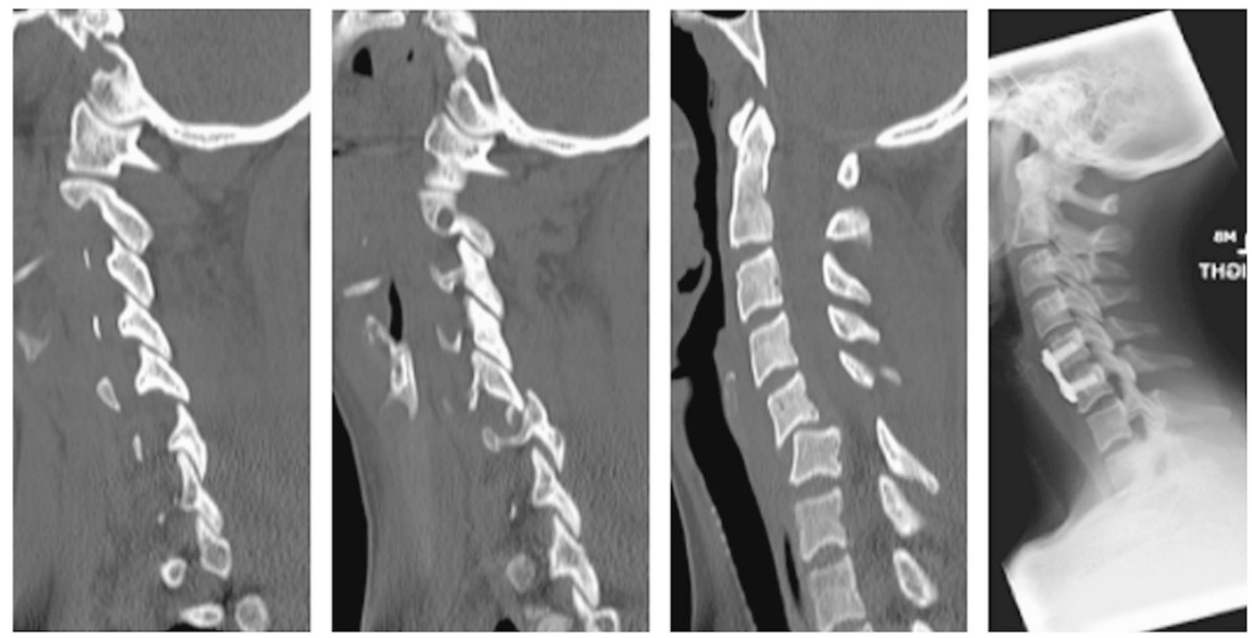

Figure 4 This patient sustained on 10 January 2013 a cervical dislocation at C5-6 as a result of a high-speed motor vehicle accident. Initial treatment involved a closed reduction using tong traction in emergency (2230 -2330 hours). Subsequent surgery was performed the next day (0800-1200 hours), with no bed rest or external immobilization prescribed. This would be coded as shown in Appendix D.

visualizes the vertebral body or inter-vertebral disk, such as thoracotomy, thoracoscopy, thoracoplasty, thoraco-abdominal, retro-peritoneal, trans-peritoneal or variations of lateral lumbar approaches. A posterior approach includes midline posterior incisions, paraspinal and Wiltse-type incisions that expose the lamina, facet joints and/or the transverse processes. Also included in the posterior approaches are percutaneous or minimally invasive surgical techniques that enable insertion of spinal implants through stab incisions.

The code 'No' is assigned if there is no open surgical procedure performed. 'Anterior open surgical procedure' is assigned if any anterior open surgical approach is utilized. 'Posterior open surgical procedure' is assigned when a posterior approach is used for an open surgical procedure, including percutaneous posterior fixation. When both an anterior and posterior approach are used as a part of the same surgical procedure performed on the same date, then 'Both anterior and posterior open surgical procedures' code is assigned. The code 'Unknown' will be assigned when an open surgical procedure has been performed, but the surgical approach is unknown.

Date and time of the intervention completion or surgical closure This variable documents the date and the time of the completion of the intervention or the date and time of the surgical closure and is recorded in a format similar to the 'Date and Time of the intervention/ procedure'. We acknowledge that a surgical procedure may begin on one day and then be completed on the same or another day. The date is recorded in standard Year/Month/Day format, and the time is recorded using the 24-h clock. Partial information can be entered. The data variable 'Unknown' is selected if there is no information available. For non-surgical interventions such as traction, closed manipulations 
or prescribed bed rest, the time data element may be left unknown as it may not be necessary.

\section{Surgical procedure-open reduction}

This variable is used to document whether or not, during the performance of an open surgical procedure the spinal elements have been manipulated to effect a reduction of a displaced fracture, a subluxation or a dislocation. Most commonly this is performed through a posterior surgical approach, but may on occasion, such as the anterior cervical discectomy followed by a reduction of a facet dislocation, be performed through an anterior approach.

The code 'No' indicates that no open reduction has been performed; 'Yes' indicates that as a component of this surgical procedure an open reduction has been performed to reduce a malalignment, subluxation or dislocation. When there has been an open surgical procedure performed but not known whether or not it was an open reduction, then 'Unknown' is assigned.

\section{Surgical procedure-direct decompression of neural elements}

This variable documents whether there has been direct surgical decompression of neural elements by removing bone, disk, ligaments, soft tissue, hematoma or foreign bodies (bullet fragments, blades or other foreign matter) from adjacent to or within the dura mater. This includes removing bone or disk that may be compressing one or more nerve roots. Direct surgical decompression does NOT include the indirect reduction that occurs with an open or closed reduction of neural elements, which may restore the spinal canal dimensions or restore the anatomy of neural foraminae. This may be performed through either an anterior or posterior surgical approach but requires direct visualization of the neural elements (dura). As such, it cannot be performed percutaneously.

The code 'No' indicates that no direct decompression of neural elements (spinal cord, cauda equina or individual nerve roots) has been performed; whereas 'Yes,' indicates that yes a direct surgical decompression has been performed through either an anterior or posterior approach or a combination of both. When the patient has had a surgical procedure and whether or not a direct surgical decompression of neural elements has been performed is not known, 'Unknown' is assigned.

\section{Surgical procedure - stabilization and fusion-segment number} This variable documents whether or not there has been an open stabilization and/or fusion procedure. When a surgical procedure is performed it may or may not include the insertion of instrumentation to stabilize vertebral levels and may or may not involve a formal fusion procedure with or without insertion of local or distantly harvested structural or non-structural bone graft or substitutes. This variable encompasses any and all of the above in one single data point.

This variable orders and assigns numbers to each region of the spine that has undergone a spinal instrumentation or fusion procedure beginning with the most cephalic region that has been instrumented and fused and progressing to the most caudal fused region regardless of which level of spinal column injury is the most severe or significant injury or which injury may or may not contribute to the patient's neurological injury. If a patient has had a vC5-6 anterior fusion and also a vT12-vL2 posterior instrumentation, then each of these noncontiguous instrumented and fused regions would receive a numerical score beginning with 1 for the vC5-6 fusion and 2 for the vT12-vL2 instrumentation.

A numerical value of ' 1 ' is assigned if only one segment of the spine has undergone instrumentation and fusion. A numerical value of ' 1 ' is also assigned to indicate the most cephalic of two or more spinal regions that have undergone fusion and/or instrumentation; ' 2 ' is assigned to the second most cephalic fused or instrumented segments; ' 3 ', ' 4 ' and so on are assigned values for three or four discrete segments of the spinal column that have been fused and/or instrumented with each number assigned in a progressive cephalic to caudal direction. The code 'Unknown' will be assigned when the order, assignment of instrumented and fused spinal segments is unknown.

\section{Surgical procedure - stabilization and fusion-segment level}

This variable is used to document each of the level(s) of spinal fusion or instrumentation. For a single fused motion segment injury, such as a C5-6 anterior fusion and plating, both the cephalic and caudal of the two adjacent vertebrae are identified and separated by a dash (-).

The prefix ' $v$ ' references the fact that we are referring to a spinal column level (vertebral level) and helps distinguish the spinal column injury level from the neurological level. The cervical region is represented by $\mathrm{vC} 00-\mathrm{vC} 07$. The occiput is considered to be the most cephalic vertebral level and is designated vC00. The thoracic region is represented by vT01-vT12. The lumbar region is represented by vL01-vL05. The sacrum is considered to be the most caudal vertebral level and is designated vS01-vS05. When the specific spinal column level is not known but the spinal region (cervical, thoracic, lumbar and sacral) is known, the regions may be represented by vC99, vT99, vL99 and vS99 for cervical, thoracic, lumbar and sacral, respectively. When both the spinal column level and the region of the spine are not known, then these levels are documented as vX99. Where there are multiple spinal column injuries, separate entries will be completed for each level of spinal column injury.

\section{DISCUSSION}

This paper describes the development of an International SCI Spinal Intervention and Surgical Procedure Basic Data Set, which is meant to accompany the International SCI Spinal Column Injury Basic Data Set, ${ }^{26}$ as well as other International SCI Data Sets. ${ }^{27}$

Although there are a number of existing classifications for spinal column procedures, ${ }^{11,28-30}$ the International SCI Spinal Intervention and Surgical Procedure Basic Data Set was developed to collect minimal amount of data and still allow comparison of data among studies, centers and countries. Interventions and procedures are most frequently described utilizing administrative variables that are ultimately used for purposes of billing for the procedures and interventions. As a result, the billing procedural codes are meant to represent how much the physician should be paid and lack the universality and precision of this set of intervention and procedure codes. This data set is produced with the goal of assisting clinicians and investigators to better compare therapeutic interventions and the timing of their application, both of which are felt to be of substantial influences on the patients' ultimate outcome.

Given that the goal is to collect a minimal amount of information on spinal interventions and procedures worldwide, there may be a need to supplement this data set with other information, such as details on the type of fusion to address specific clinical topics or answer research questions. As the International SCI Spinal Intervention and Surgical Procedure Basic Data Set is used by clinicians and researches, it may identify gaps that could inform a future revision to the existing data set or justify the development of an expanded data set similar to other areas, such as bowel. ${ }^{31}$

With the high frequency of reported adverse events and the associated costs that occur with the provision of care, individuals using this data set may want to also collect data on intra-operative 
adverse events such as airway/ventilation complications, hardware malposition or a dural tear. A proposed list of important intraoperative adverse events was first described in the Spine Adverse Events Severity System in patients undergoing spine procedures and later used in patients with traumatic SCI. ${ }^{32-35} \mathrm{~A}$ recent study by Street et al. ${ }^{34}$ reported that $14.6 \%$ of patients with traumatic SCI experience at least one intra-operative complication, with dural tear, anestheticrelated complications, blood loss and hardware malposition requiring revision being the most common. Supplementing the International SCI Spinal Intervention and Surgical Procedure Basic Data Set with this type of data will further elucidate the appropriateness of the various interventions and procedures for specific injury morphologies (for example, effectiveness of closed reductions or the optimal surgical approach for stabilizing cervical facet fractures) or patient characteristics (for example, elderly, multiple fractures and so on).

To assist with the global adoption of the International SCI Data Sets, there will be a need to translate the data set into other languages. It is recommended that any translation is conducted in a systematic and rigorous manner as outlined by Biering-Sorensen et al. ${ }^{36}$ Furthermore, to assist with standardizing how data from the International SCI Core Data Set are reported, guidelines have been published, ${ }^{25}$ and similar initiatives for both the International SCI Spinal Column Injury and the Spinal Interventions and Surgical Procedures Basic Data Sets should be considered in the future. The incorporation of the International SCI Data Sets as part of the National Institute of Neurological Disorders and Stroke Common Data Element project ${ }^{37}$ will facilitate the incorporation of these data elements into future studies and provide guidance on how best to revise and report on these data elements.

In conclusion, this paper describes the development of the International SCI Spinal Intervention and Surgical Procedure Injury Basic Data Set that was developed and peer-reviewed with input from International experts. The nine data elements included in this data set encompasses the most relevant information on spinal interventions and procedures. This data set was developed to facilitate the collection of a minimal amount of information on the spinal interventions and surgical procedures for patients sustaining a traumatic SCI. Collection of this information will start to standardize the reporting and facilitate research worldwide. Feedback from users of this existing data set as well as recommendations from new initiatives will inform future revisions to the International SCI Spinal Intervention and Surgical Procedure Injury Basic Data Set.

\section{DATA ARCHIVING}

There were no data to deposit.

\section{CONFLICT OF INTEREST}

Dr Michael G Fehlings and Dr Marcel F Dvorak have received funding from the Rick Hansen Institute. Dr Vanessa K Noonan is an employee of the Rick Hansen Institute. The remaining authors declare no conflict of interest.

\section{ACKNOWLEDGEMENTS}

Funding and in-kind support was provided by the Rick Hansen Foundation and the Rick Hansen Institute, respectively. We are thankful for comments and suggestions received from Susan Charlifue, Lawrence Vogel, Marcel Post, Marcalee Sipski Alexander, Andrew Nataraj, Richard Fox, Inge Eriks-Hoogland and Sonja Meier. In addition, we would like to thank Sophia Park for her assistance in preparing this document.
1 Gelb DE, Aarabi B, Dhall SS, Hurlbert RJ, Rozzelle CJ, Ryken TC et al. Treatment of subaxial cervical spinal injuries. Neurosurgery 2013; 72 (Suppl 2): 187-194.

2 Ryken TC, Aarabi B, Dhall SS, Gelb DE, Hurlbert RJ, Rozzelle CJ et al. Management of isolated fractures of the atlas in adults. Neurosurgery 2013; 72 (Suppl 2): 127-131.

3 Katoh S, el Masry WS, Jaffray D, McCall IW, Eisenstein SM, Pringle RG et al. Neurologic outcome in conservatively treated patients with incomplete closed traumatic cervical spinal cord injuries. Spine 1996; 21: 2345-2351.

4 Wilson JR, Singh A, Craven C, Verrier MC, Drew B, Ahn H et al. Early versus late surgery for traumatic spinal cord injury: the results of a prospective Canadian cohort study. Spinal Cord 2012; 50: 840-843.

5 Fehlings MG, Vaccaro A, Wilson JR, Singh AW, Cadotte D, Harrop JS et al. Early versus delayed decompression for traumatic cervical spinal cord injury: results of the Surgical Timing in Acute Spinal Cord Injury Study (STASCIS). PLoS One 2012; 7: e32037.

6 Chipman JG, Deuser WE, Beilman GJ. Early surgery for thoracolumbar spine injuries decreases complications. J Trauma 2004; 56: 52-57.

7 Tator CH, Fehlings MG, Thorpe K, Taylor W. Current use and timing of spinal surgery for management of acute spinal cord injury in North America: results of a retrospective multicenter study. J Neurosurg 1999; 91 (Suppl 1): 12-18.

8 Biering-Sorensen F, Charlifue S, DeVivo M, Noonan V, Post M, Stripling T et al. International Spinal Cord Injury Datasets. Spinal Cord 2006; 44: 530-534.

9 Levine AM, Edwards CC. Fractures of the atlas. J Bone Joint Surg Am 1991; 73 680-691.

10 Kanter AS, Wang MY, Mummaneni PV. A treatment algorithm for the management of cervical spine fractures and deformity in patients with ankylosing spondylitis. Neurosurg Focus 2008; 24: E11.

11 Patel AA, Hurlbert RJ, Bono CM, Bessey JT, Yang N, Vaccaro AR. Classification and surgical decision making in acute subaxial cervical spine trauma. Spine 2010; 35 (21 Suppl): S228-S234.

12 Gelb DE, Hadley MN, Aarabi B, Dhall SS, Hurlbert RJ, Rozzelle CJ et al. Initial closed reduction of cervical spinal fracture-dislocation injuries. Neurosurgery 2013; 72 (Suppl 2): 73-83.

13 Keynan O, Dvorak M, Fisher C. Reduction techniques in cervical facet dislocations. Tech Orthop 2002; 17: 336-344.

14 Thomas KC, Bailey CS, Dvorak MF, Kwon B, Fisher C. Comparison of operative and nonoperative treatment for thoracolumbar burst fractures in patients without neurological deficit: a systematic review. J Neurosurg Spine 2006; 4: 351-358.

15 Bailey CS, Dvorak MF, Thomas KC, Boyd MC, Paquett S, Kwon BK et al. Comparison of thoracolumbosacral orthosis and no orthosis for the treatment of thoracolumbar burst fractures: interim analysis of a multicenter randomized clinical equivalence trial. $J$ Neurosurg Spine 2009; 11: 295-303.

16 Katoh S, el Masry WS. Motor recovery of patients presenting with motor paralysis and sensory sparing following cervical spinal cord injuries. Paraplegia 1995; 33: 506-509.

17 el Masry WS.. Physiological instability of the spinal cord following injury. Paraplegia 1993; 31: 273-275.

18 Rampersaud YR, Annand N, Dekutoski MB. Use of minimally invasive surgical techniques in the management of thoracolumbar trauma: current concepts. Spine 2006; 31 (11 Suppl): S96-S102; discussion S104.

19 Remes V, Lamberg T, Tervahartiala P, Helenius I, Schlenzka D, Yrjonen T et al. Longterm outcome after posterolateral, anterior, and circumferential fusion for high-grade isthmic spondylolisthesis in children and adolescents: magnetic resonance imaging findings after average of 17-year follow-up. Spine 2006; 31: 2491-2499.

20 Clohisy JC, Akbarnia BA, Bucholz RD, Burkus JK, Backer RJ. Neurologic recovery associated with anterior decompression of spine fractures at the thoracolumbar junction (T12-L1). Spine 1992; 17 (8 Suppl): S325-S330.

21 Verlaan JJ, Diekerhof CH, Buskens E, van der Tweel I, Verbout AJ, Dhert WJ et al. Surgical treatment of traumatic fractures of the thoracic and lumbar spine: a systematic review of the literature on techniques, complications, and outcome. Spine 2004; 29 803-814

22 Dvorak MF, Fisher CG, Fehlings MG, Rampersaud YR, Oner FC, Aarabi B et al. The surgical approach to subaxial cervical spine injuries: an evidence-based algorithm based on the SLIC classification system. Spine 2007; 32: 2620-2629.

23 Biering-Sorensen F, Bickenbach JE, EI Masry WS, Officer A, von Groote PM. ISCoSWHO collaboration. International Perspectives of Spinal Cord Injury (IPSCI) report. Spinal Cord 2011; 49: 679-683.

24 DeVivo M, Biering-Sorensen F, Charlifue S, Noonan V, Post M, Stripling T. et al International Spinal Cord Injury Core Dataset. Spinal Cord 2006; 44: 535-540.

25 DeVivo MJ, Biering-Sorensen F, New P, Chen Y. Standardization of data analysis and reporting of results from the International Spinal Cord Injury Core Dataset. Spinal Cord 2011; 49: 596-599.

26 Dvorak MF, Wing PC, Fehlings MG, Vaccaro AR, Itshayek E, Biering-Sorensen F et al. International Spinal Cord Injury Spinal Column Injury Basic Dataset. Spinal Cord 2012 50: 817-821.

27 International Spinal Cord Society. International SCI Datasets. Available at: http://www. iscos.org.uk/international-sci-data-sets. 04 Feb 2014.

28 Allen BL Jr, Ferguson RL, Lehmann TR, O'Brien RP.. A mechanistic classification of closed, indirect fractures and dislocations of the lower cervical spine. Spine 1982; 7: $1-27$

29 Aarabi B, Walters BC, Dhall SS, Gelb DE, Hurlbert RJ, Rozzelle CJ et al. Subaxial cervical spine injury classification systems. Neurosurgery 2013; 72 (Suppl 2): $170-186$ 
30 Vaccaro AR, Oner C, Kepler CK, Dvorak M, Schnake K, Bellabarba C et al. AOSpine thoracolumbar spine injury classification system: fracture description, neurological status, and key modifiers. Spine 2013; 38: 2028-2037.

31 Krogh K, Perkash I, Stiens SA, Biering-Sorensen F. International bowel function extended spinal cord injury dataset. Spinal Cord 2009; 47: 235-241.

32 Street JT, Noonan VK, Cheung A, Fisher CG, Dvorak MF.. Incidence of acute care adverse events and long-term health related quality of life in patients with traumatic spinal cord injury. Spine 2013; 51: 472-476.

33 Street JT, Thorogood NP, Cheung A, Noonan VK, Chen J, Fisher CG et al. Use of the Spine Adverse Events Severity System (SAVES) in patients with traumatic spinal cord injury. A comparison with institutional ICD-10 coding for the identification of acute care adverse events. Spinal Cord 2013; 51: 472-476.
34 Street JT, Lenehan BJ, DiPaola CP, Boyd MD, Kwon BK, Paquette SJ et al. Morbidity and mortality of major adult spinal surgery. A prospective cohort analysis of 942 consecutive patients. Spine 2012; 12: 22-34.

35 Rampersaud YR, Moro ER, Neary MA, White K, Lewis SJ, Massicotte EM et al. Intraoperative adverse events and related postoperative complications in spine surgery: implications for enhancing patient safety founded on evidence-based protocols. Spine 2006; 31: 1503-1510.

36 Biering-Sorensen F, Alexander MS, Burns S, Charlifue S, DeVivo M, Dietz V et al. Recommendations for translation and reliability testing of International Spinal Cord Injury Datasets. Spinal Cord 2011; 49: 357-360.

37 Biering-Sorensen F, Charlifue S, Devivo MJ, Grinnon ST, Kleitman N, Lu Y et al. Incorporation of the International Spinal Cord Injury Dataset elements into the National Institute of Neurological Disorders and Stroke Common Data Elements. Spinal Cord 2011; 49: 60-64.

Supplementary Information accompanies this paper on the Spinal Cord website (http://www.nature.com/sc)

\section{APPENDIX A}

Intervention/procedure date and start time:
2010/02/06;
1630 hours;
Unknown

Non-surgical bed rest and external immobilization:

$\square$ No

$\square$ Enforced bed rest

$\mathbf{X}$ External immobilizing device

$\square$ Both enforced bed rest and external immobilizing device

$\square$ Unknown

Spinal intervention—closed manipulation and/or reduction of spinal elements:

$X$ No

$\square$ Yes

$\square$ Unknown

Surgical procedure-approach:

$\square$ No

$\mathrm{X}$ Anterior open surgical procedure

$\square$ Posterior open surgical procedure

$\square$ Both anterior and posterior open surgical procedures

Unknown

Date and time of the intervention completion or surgical closure:

2010/02/06; 2245 hours; $\square$ Unknown

If an open surgical approach was performed (anterior, posterior or both anterior and posterior), fill in the data elements below.

\section{Surgical procedure-open reduction:}

$\square$ No

$\mathrm{X}$ Yes

$\square$ Unknown

Surgical procedure-direct decompression of neural elements:

$\square$ No

$\mathbf{X}$ Yes

$\square$ Unknown

Surgical procedure-stabilization and fusion: (one to be filled in for each level of injury, starting with the most cephalic injury): Stabilization and fusion-segment number: 1

Stabilization and fusion-segment level: vC02

Stabilization and fusion-segment number: 2

Stabilization and fusion-segment level: vC05-vC07 
vC00-vC07-Cervical (C0-C07) vC99-Unknown cervical (C0-C07)

vT01-vT12-Thoracic (T1-T12) vT99-Unknown thoracic (T1-T12)

vL01-vL05-Lumbar (L1-L5) vL99-Unknown lumbar (L1-L5)

vS01-vS05-Sacral (S1-S5) vS99-Unknown sacral (S1-S5)

vX99-Unknown level

\section{APPENDIX B}

Intervention/procedure date and start time:
2011/06/26;
2300 hours;
Unknown

Non-surgical bed rest and external immobilization:

$\mathrm{X}$ No

$\square$ Enforced bed rest

$\square$ External immobilizing device

$\square$ Both enforced bed rest and external immobilizing device

$\square$ Unknown

Spinal intervention-closed manipulation and/or reduction of spinal elements:

$\mathrm{X}$ No

$\square$ Yes

$\square$ Unknown

Surgical procedure-approach:

$\square$ No

$\square$ Anterior open surgical procedure

$\square$ Posterior open surgical procedure

$\mathbf{X}$ Both anterior and posterior open surgical procedures

$\square$ Unknown

Date and time of the intervention completion or surgical closure:

2011/06/27; 0430 hours; $\square$ Unknown

If an open surgical approach was performed (anterior, posterior or both anterior and posterior), fill in the data elements below.

Surgical procedure-open reduction:

$X$ No

$\square$ Yes

$\square$ Unknown

Surgical procedure-direct decompression of neural elements:

$\square$ No

$\mathbf{X}$ Yes

$\square$ Unknown

Surgical procedure-stabilization and fusion: (one to be filled in for each level of injury, starting with the most cephalic injury): Stabilization and fusion-segment number: 1

Stabilization and fusion-segment level: vT11-vL2

vC00-vC07-Cervical (C0-C07) vC99-Unknown cervical (C0-C07)

vT01-vT12-Thoracic (T1-T12) vT99-Unknown thoracic (T1-T12)

vL01-vL05-Lumbar (L1-L5) vL99-Unknown lumbar (L1-L5)

vS01-vS05-Sacral (S1-S5) vS99-Unknown sacral (S1-S5)

vX99-Unknown level 


\section{APPENDIX C}

Intervention/procedure date and start time:

2012/08/15;

time unknown;

Unknown

Non-surgical bed rest and external immobilization:

$\square$ No

$\mathbf{X}$ Enforced bed rest

$\square$ External immobilizing device

$\square$ Both enforced bed rest and external immobilizing device

$\square$ Unknown

Spinal intervention—closed manipulation and/or reduction of spinal elements:

$\mathrm{X}$ No

$\square$ Yes

$\square$ Unknown

Surgical procedure-approach:

$X$ No

$\square$ Anterior open surgical procedure

$\square$ Posterior open surgical procedure

$\square$ Both anterior and posterior open surgical procedures

$\square$ Unknown

Date and time of the intervention completion or surgical closure:

2012/09/05;

time unknown;

$\square$ Unknown

If an open surgical approach was performed (anterior, posterior or both anterior and posterior), fill in the data elements below.

Surgical procedure-open reduction:

$\square$ No

$\square$ Yes

$\square$ Unknown

Surgical procedure- direct decompression of neural elements:
$\square$ No
$\square$ Yes
$\square$ Unknown

Surgical procedure-stabilization and fusion: (one to be filled in for each level of injury, starting with the most cephalic injury): Stabilization and fusion-segment number

Stabilization and fusion—segment level

vC00-vC07-Cervical (C0-C07) vC99-Unknown cervical (C0-C07)

vT01-vT12-Thoracic (T1-T12) vT99-Unknown thoracic (T1-T12)

vL01-vL05-Lumbar (L1-L5) vL99-Unknown lumbar (L1-L5)

vS01-vS05-Sacral (S1-S5) vS99-Unknown sacral (S1-S5)

vX99-Unknown level 


\section{APPENDIX D}

Intervention/procedure date and start time:
2013/01/10;
2230 hours;
Unknown

Non-surgical bed rest and external immobilization:

$\mathrm{X}$ No

$\square$ Enforced bed rest

$\square$ External immobilizing device

$\square$ Both enforced bed rest and external immobilizing device

$\square$ Unknown

Spinal intervention—closed manipulation and/or reduction of spinal elements:
$\square$ No
$\mathrm{X}$ Yes
$\square$ Unknown

Surgical procedure-approach:

$\mathrm{X}$ No

$\square$ Anterior open surgical procedure

$\square$ Posterior open surgical procedure

$\square$ Both anterior and posterior open surgical procedures

$\square$ Unknown

Date and time of the intervention completion or surgical closure:
2013/01/10;
2330 hours;
$\square$ Unknown

If an open surgical approach was performed (anterior, posterior or both anterior and posterior), fill in the data elements below. Surgical procedure-open reduction:
$\square$ No
$\square$ Yes
$\square$ Unknown

Surgical procedure-direct decompression of neural elements:

$\square$ No
$\square$ Yes
$\square$ Unknown

Surgical procedure-stabilization and fusion: (one to be filled in for each level of injury, starting with the most cephalic injury): Stabilization and fusion-segment number _ -

Stabilization and fusion-segment level_

vC00-vC07-Cervical (C0-C07) vC99-Unknown cervical (C0-C07)

vT01-vT12-Thoracic (T1-T12) vT99-Unknown thoracic (T1-T12)

vL01-vL05-Lumbar (L1-L5) vL99-Unknown lumbar (L1-L5)

vS01-vS05-Sacral (S1-S5) vS99-Unknown sacral (S1-S5)

vX99-Unknown level

Intervention/procedure date and start time:

2013/01/11; 0800 hours; $\square$ Unknown

Non-surgical bed rest and external immobilization:

$\mathrm{X}$ No

$\square$ Enforced bed rest

$\square$ External immobilizing device

$\square$ Both enforced bed rest and external immobilizing device

$\square$ Unknown

Spinal intervention-closed manipulation and/or reduction of spinal elements:

$\mathrm{X}$ No

$\square$ Yes

$\square$ Unknown 
Surgical procedure-approach:

$\square$ No

$\mathbf{X}$ Anterior open surgical procedure

$\square$ Posterior open surgical procedure

$\square$ Both anterior and posterior open surgical procedures

$\square$ Unknown

Date and time of the intervention completion or surgical closure:

2013/01/11; 1200 hours; $\square$ Unknown

If an open surgical approach was performed (anterior, posterior or both anterior and posterior), fill in the data elements below.

Surgical procedure-open reduction:

$\mathrm{X}$ No

$\square$ Yes

$\square$ Unknown

Surgical procedure-direct decompression of neural elements:

$\square$ No

X Yes

$\square$ Unknown

Surgical procedure-stabilization and fusion: (one to be filled in for each level of injury, starting with the most cephalic injury): Stabilization and fusion-segment number: 1

Stabilization and fusion-segment level: vC05-vC06

vC00-vC07-Cervical (C0-C07) vC99-Unknown cervical (C0-C07)

vT01-vT12-Thoracic (T1-T12) vT99-Unknown thoracic (T1-T12)

vL01-vL05-Lumbar (L1-L5) vL99-Unknown lumbar (L1-L5)

vS01-vS05-Sacral (S1-S5) vS99-Unknown sacral (S1-S5)

vX99-Unknown level 